\title{
Muscle Mitochondrial Dysfunction in Horses Affected by Acute Laminitis
}

Didier Serteyn ${ }^{1-3^{*}}$, Geoffroy de la Rebière de Pouyade ${ }^{1,2}$, Charlotte Sandersen ${ }^{1,2}$, Alexandra Salciccia ${ }^{1}$, Sigrid Grulke ${ }^{1}$, Ange Mouithys-Mickalad ${ }^{2}$, Thierry Franck $^{2}$, Jean-Philippe Lejeune ${ }^{1,3}$ and Justine Ceusters ${ }^{2}$

${ }^{1}$ Department of Clinical Sciences, Equine Clinic, Faculty of Veterinary Medicine B41, University of Liege, 4000 Sart Tilman, Belgium

${ }^{2}$ Centre for Oxygen Research and Development, B6a, University of Liege, 4000 Sart Tilman, 4000, Belgium

${ }^{3}$ Equine Research Centre, Mont-le-Soie, 6690 Vielsalm, Belgium

"Corresponding author: Didier Serteyn, Department of Clinical Sciences, Equine Clinic, Faculty of Veterinary Medicine B41, University of Liege, 4000 Sart Tilman, Belgium, Tel: +32 4-366-41-03; E-mail: didier.serteyn@ulg.ac.be

Received date: July 16, 2014; Accepted date: September 29, 2014, Published date: October 6, 2014

Copyright: $\odot 2014$ Serteyn D, et al. This is an open-access article distributed under the terms of the Creative Commons Attribution License, which permits unrestricted use, distribution, and reproduction in any medium, provided the original author and source are credited.

\begin{abstract}
Laminitis is a common and debilitating disease affecting horses and ponies. It often leads to the demise of the animal. Energy deficiency is suspected to entrain the disruption of the hemidesmosomes leading to the failure of the dermal-epidermal interface. The aim of this study was to measure the muscle mitochondrial function by high resolution respirometry. Muscle micro-biopsies were obtained from 11 horses affected by acute metabolic laminitis, 6 horses affected by acute laminitis resulting from a systemic inflammation response syndrome and 28 healthy horses distributed in 2 control groups: 17 horses with a body condition score [BSC, ranging from 0 (emaciated) to 5 (obese)] of 2 to 3 and 11 horses with a BSC of 4 to 5 . During the acute phase of laminitis, a significant reduction of the muscle mitochondrial respiration was observed. The muscle mitochondrial dysfunction occurred independently of the etiology (metabolic disorder or systemic inflammation) leading to laminitis. The reduction of the oxidative phosphorylation and of the maximal respiratory capacity (after uncoupling) may induce depletion of the cell's ATP content. If the same mitochondrial alteration occurs in the foot lamina, mitochondria targeting should be considered for the future, not only to better understand the physiopathology of the disease but also to maintain and to support the mitochondrial function before reaching the " mitochondrial dysfunction threshold » that may lead to the failure of the dermal-epidermal interface.
\end{abstract}

Keywords: Horse; Laminitis; Mitochondria; Electron transfer system; Energy metabolism dysfunction; Oxidative phosphorylation; Inflammation; Reactive oxygen species

\section{Introduction}

Laminitis is a common and debilitating disease affecting horses and ponies. In its severe form it may necessitate the demise of the animal [1]. Laminitis is characterized by the failure of the laminar dermalepidermal interface resulting in severe lameness.

Despite ongoing research, laminitis remains a severely damaging condition of which the physiopathology is poorly understood. Currently, the primary hypotheses explaining the pathogenesis of acute laminitis include inflammation and extracellular matrix degradation, metabolic abnormalities and endothelial/vascular dysfunction [2].

Recent epidemiological studies have identified a variety of risk factors for the development of laminitis, such as weight gain, season of the year, hours of sunshine on the pasture, recent access to grass, box rest, owner-reported history of laminitis, lameness or foot-soreness after shoeing/trimming, and existing endocrinopathic and metabolic disease [3,4]. Endotoxemia and sepsis have been recognized in a follow-up of hospitalized horses as the only significant risk factor for developing laminitis [5]. Inflammation seems to play a central role in the pathogenesis of laminitis, and this independently of the key factors involved [6]. In cases of sepsis, local infection is accompanied by systemic neutrophil activation. Systemic neutrophil activation is also encountered in equine laminitis, as demonstrated by the up-regulation of cytokine expression, the dynamic changes in blood neutrophil phenotype, the formation of neutrophil-platelet aggregates and the infiltration of inflammatory cells [7-10]. However, other factors than endotoxins are responsible for the changes in laminar tissue gene expression that occurs during the development of acute equine laminitis [11,12].

The systemic and the local inflammatory responses and the release of cytokines, enzymes such as myeloperoxidase or elastase, have largely been described in previously developed laminitis models such as Carbohydrate Overload (CHO), Oligofructose (OF) and Black Walnut Heartwood Extract (BWHE). This underlines the major role of neutrophil activation [13-16]. The hypothesis of an involvement of neutrophil-mediated mitochondrial dysfunction was previously observed in muscle micro-biopsies from horses after strenuous exercise [17]. Moreover, in cultured muscle cell lines, it was demonstrated that the potent neutrophil-derived oxidative enzyme myeloperoxidase could enter the cell and disturb the electron transfer system resulting in altered ATP production [18].

Laminitis has also been associated with horses diagnosed with metabolic disorders, such as obesity, pituitary pars intermedia dysfunction or equine metabolic syndrome. The primary characteristic of these pathologies is the development of insulin resistance, characterized by hyperinsulinemia with eu- or hyperglycemia and subsequent chronic pro-inflammatory state $[19,20]$. While several aspects of the relationship between insulin resistance and laminitis have been investigated, the underlying pathological pathway has not yet been identified. An experimental model of laminitis showed that healthy Standard bred horses subjected to prolonged 
Page 2 of 7

hyperinsulinaemia develops laminitis within $48 \mathrm{~h}$ [21]. Furthermore, natural cases of equine endocrinopathic laminitis are clearly associated with hyperinsulinemia [22].

Recently, Gauff et al. showed that short-term hyperinsulinaemia in the isolated, perfused extracorporeal equine digit leads to a marked increase in vascular resistance and an increase in endothelin-1 expression [23].

Several studies confirmed a mitochondrial dysfunction in human patients suffering from metabolic disorders related to insulin resistance [24,25]. Indeed, glucose and lipid metabolism are largely dependent on energy generated by mitochondria and several experimental protocols have demonstrated an effect of hyperinsulinemia on mitochondrial function $[26,27]$.

In this work, we hypothesized that muscle mitochondrial function is altered in horses suffering from laminitis. As the mitochondrial function is frequently studied in muscles, we decided to investigate this using High-Resolution Respirometry (HRR) on muscular microbiopsies [28]. High-resolution respirometry allows the determination of Oxidative Phosphorylation (OXPHOS) and Electron Transfer System (ETS) capacities in small samples $(2 \mathrm{mg})$ of permeabilized tissues [28]. Multiple Substrate-Uncoupler-Inhibitor Titration (SUIT) protocols have been recently developed to screen the mitochondrial function of equine muscles [29]. In the present study, this technique was used to study mitochondrial function in laminitis affected horses.

\section{Material and Method}

\section{Horses}

All procedures were approved by the Animal Ethic Commission of the University of Liege (agreement $n^{\circ} 07-629$ ) and informed owner consent was obtained when appropriate. Muscle micro-biopsy is performed on healthy horses $(n=28)$ and on clinical cases of acute laminitis observed in the Equine Clinic of the University of Liege between 2011 and $2013(\mathrm{n}=17)$. The Body Condition Score (BSC) was determined based on palpation of fat deposits in five specific body areas and by visual assessment of seven anatomic sites [30]. Each evaluation receives a notation and the average defines the BSC ranging from 0 (emaciated) to 5 (obese). All the horses were categorized in two groups: score 0 for a BSC between 2 to $3(n=23)$ and score 1 for a BSC between 4 to $5(n=22)$.

The healthy horses were divided in two control groups: control obese (contob) and control non obese (cont fit). Muscle microbiopsies were obtained from 2 distinct groups of acute laminitic horses. The first group was assigned as "metabolic laminitis" (Met Lam). These horses were showing symptoms of acute laminitis and were diagnosed as suffering from metabolic syndrome [31]. The second group was assigned as "inflammatory laminitis" (Infl Lam) and was composed of horses affected by acute laminitis resulting from a systemic inflammatory response syndrome.

So, 4 groups of horses were established: healthy non obese (Cont fit: $\mathrm{n}=17$ ), healthy obese (Contob: $\mathrm{n}=11$ ), laminitic obese (Met Lam, $\mathrm{n}=11$ ) and laminitic non obese (Infl Lam: $n=6$ ). All data obtained from the horses are reported in the Table 1.

\section{Muscle micro-biopsy}

Approximately $20 \mathrm{mg}$ of muscle have been collected from the triceps brachii muscle using a $14 \mathrm{G}$ biopsy needle mounted on an automatic instrument as earlier described (Pro-Mag ${ }^{\text {ma }}$ Ultra Biopsy Instrument, Angiotech, Gainesville, Fl, USA) [29]. Briefly, the sampling site was shaved and desensitized by subcutaneous injection of $0.5 \mathrm{ml}$ of mepivacain (Scandicaine 2\%, AstraZeneca, Brussels, Belgium), and aseptically prepared.

Muscle micro-biopsy specimens were taken at $40 \mathrm{~mm}$ depth in the long head of the triceps brachii through a skin incision. Two to three muscle samples were obtained via the same skin opening and transferred immediately into ice-cold relaxing solution BIOPS containing $10 \mathrm{mM} \mathrm{CaK}$-EGTA, $7.23 \mathrm{mM} \mathrm{K2-EGTA,} 20 \mathrm{mM}$ imidazole, $20 \mathrm{mM}$ taurine, $50 \mathrm{mM}$ K-MES, $0.5 \mathrm{mM}$ dithiothreitol, 6.56 $\mathrm{mM} \mathrm{MgCl} 2,5.77 \mathrm{mM}$ ATP and $15 \mathrm{mM}$ phosphocreatine adjusted to $\mathrm{pH}$ 7.1. Fibers were kept at $4^{\circ} \mathrm{C}$ until further preparation.

\begin{tabular}{|c|c|c|c|c|c|c|c|}
\hline & Age & Weight & BCS & $\mathrm{Cl}$ & $\mathrm{Cl}+\mathrm{II}$ & $\mathbf{E T S}_{\max }$ & ClI \\
\hline Metabolic laminitis (EMS) & (Years) & $(\mathrm{Kg})$ & $(/ 5)$ & \multicolumn{4}{|c|}{ (pmol oxygen/s* mg muscle) } \\
\hline HoM1 & 12 & 625 & 4 & 34.46 & 75.5 & 83.38 & 58.28 \\
\hline HoM2 & 17 & 406 & 4 & 36.66 & 96.76 & 111.72 & 75.94 \\
\hline HoM3 & 12 & 475 & 5 & 42.09 & 67.75 & 91.42 & 53.52 \\
\hline HoM4 & 16 & 590 & 4 & 55.08 & 96.56 & 129.90 & 80.99 \\
\hline HoM5 & 23 & 310 & 4 & 36.34 & 64.87 & 82.43 & 54.56 \\
\hline HoM6 & 11 & NA & 4 & 56.05 & 89.07 & 138.06 & 73.58 \\
\hline HoM7 & 11 & NA & 4 & 47.35 & 94.23 & 127.73 & 82.34 \\
\hline HoM8 & 13 & 683 & 5 & 30.69 & 46.80 & 72.84 & 43.86 \\
\hline HoM9 & 4 & 650 & 4 & 29.25 & 45.16 & 62.45 & 37.25 \\
\hline HoM10 & 16 & 620 & 4 & 49.31 & 78.72 & 96.69 & 56.93 \\
\hline HoM11 & 8 & 530 & 4 & 39.28 & 61.94 & 79.57 & 47.09 \\
\hline
\end{tabular}


Page 3 of 7

\begin{tabular}{|c|c|c|c|c|c|c|c|}
\hline mean $( \pm S D)$ & $13 \pm 4$ & $522 \pm 122$ & $4-5$ & $45.73 \pm 17.11$ & $82.38 \pm 33.23$ & $108.65 \pm 44.58$ & $68.31 \pm 31.17$ \\
\hline \multicolumn{8}{|c|}{ Control obese $(n=11)$} \\
\hline Mean $( \pm S D)$ & $12 \pm 5$ & nd & $4-5$ & $51.60 \pm 13.98$ & $102.76 \pm 23.45$ & $119.79 \pm 24.27$ & $81.85 \pm 16.79$ \\
\hline \multicolumn{8}{|c|}{ Inflammatory laminitis } \\
\hline Hol1 & 10 & 625 & 3 & 27.00 & 65.50 & 70.40 & 54.60 \\
\hline $\mathrm{Hol} 2$ & 2 & 470 & 2 & 52.37 & 77.78 & 103.16 & 56.21 \\
\hline Hol3 & 3 & 700 & 3 & 27.02 & 48.17 & 73.15 & 42.67 \\
\hline $\mathrm{Hol} 4$ & 21 & 550 & 3 & 48.64 & 71.50 & 97.82 & 56.27 \\
\hline Hol5 & 11 & 580 & 2 & 78.28 & 118.38 & 151.27 & 78.80 \\
\hline Hol6 & 4 & 700 & 3 & 35.36 & 67.31 & 87.07 & 57.05 \\
\hline mean $( \pm S D)$ & $9 \pm 7$ & $604 \pm 89$ & $2-3$ & $44.78 \pm 19.57$ & $74.77 \pm 23.54$ & $97.15 \pm 29.52$ & $57.60 \pm 11.70$ \\
\hline \multicolumn{8}{|c|}{ Control non obese $(n=17)$} \\
\hline mean $( \pm \mathrm{SD})$ & $8 \pm 3$ & nd & $2-3$ & $58.79 \pm 12.6$ & $116.37 \pm 18.9$ & $139.66 \pm 19.38$ & $96.13 \pm 12.82$ \\
\hline
\end{tabular}

Table 1: Characteristics of horses used in the study and their respiratory parameters from substrate-uncoupler-inhibitor titration protocol 1 (SUIT1) applied on equine permeabilized muscle fibers of triceps brachii: Complex I (CI), Complex I + II (CI+II), maximal respiratory (ETSmax) and Complex II (CII) capacities.

\section{High resolution respirometry}

Connective tissue and other visible tissues than muscle were removed and muscle fibers were mechanically separated using two pairs of forceps with sharp tips. Complete permeabilization of the plasma membrane was obtained by gentle agitation for $30 \mathrm{~min}$ at $4^{\circ} \mathrm{C}$ in $2 \mathrm{ml}$ of BIOPS solution containing $50 \mu \mathrm{g} / \mathrm{ml}$ saponin. The fiber bundles were rinsed by agitation for $10 \mathrm{~min}$ in ice-cold mitochondrial respiration medium $\left(\mathrm{MiR0}_{5}\right.$; $0.5 \mathrm{mM}$ EGTA, $3 \mathrm{mM} \mathrm{MgCl} 2,60 \mathrm{mM} \mathrm{K}-$ lactobionate, $20 \mathrm{mM}$ taurine, $10 \mathrm{mM} \mathrm{KH}_{2} \mathrm{PO}_{4}, 20 \mathrm{mM}$ Hepes, $110 \mathrm{mM}$ sucrose and $1 \mathrm{~g} / \mathrm{l} \mathrm{BSA}$ essentially fatty acid free) adjusted to $\mathrm{pH}$ 7.1. The permeabilized muscle fibers (Pfi) were immediately used for HRR.

One to $2.5 \mathrm{mg}$ (Microbalance; Mettler Toledo, Zaventem, Belgium) of Pfi were added to each Oxygraph-2k chamber (Oroboros Instruments, Innsbruck, Austria) containing $2 \mathrm{ml}$ of MiR05 at $37.0^{\circ} \mathrm{C}$. Oxygen concentration $(\mu \mathrm{M})$, and oxygen flux per muscle mass (pmol $\mathrm{O}_{2} / \mathrm{sec}^{\star} \mathrm{mg}$ ) were recorded online using DatLab software (Oroboros Instruments, Innsbruck, Austria). After calibration of the oxygen sensors at air saturation, a few $\mathrm{ml}$ of oxygen were introduced into the gas phase above the stirred aqueous phase in the partially closed chambers to reach a concentration of $500 \mu \mathrm{M} \mathrm{O}$. During SUIT protocols (about 1 hour), the oxygen level in the chambers was maintained between 200 and $500 \mu \mathrm{M} \mathrm{O} \mathrm{O}_{2}$ to avoid any oxygen limitation of respiration. As recommended, respiratory flux was corrected on-line for instrumental background, determined at experimental oxygen levels.

To obtain extended information on coupling and substrate control parameters, the SUIT protocol was applied on Pfi. In this protocol, electron flow through complex I (CI) was supported by the NADHlinked substrates glutamate + malate $(\mathrm{GM} ; 10$ and $2 \mathrm{mM})$ with subsequent addition of ADP (2.5 mM). ADP-stimulated respiration represents OXPHOS capacity (State $\mathrm{P}$ ). Then, we added succinate (S; $10 \mathrm{mM}$ ) for convergent electron flow through complex I and II (CI+II) into the Q-junction (supported by GMS). The capacity of the phosphorylation system (adenine nucleotide translocase, inorganic phosphate transporter, and ATP synthase) may limit OXPHOS capacity with an apparent excess capacity of the electron transport system (ETS) over the phosphorylation system [28]. This was tested by stepwise addition of the uncoupler FCCP (Carbonyl cyanide 4(trifluoromethoxy)phenylhydrazone; $0.05 \mu \mathrm{M}$, followed by several $0.025 \mu \mathrm{M}$ additions (steps) until maximal oxygen flux was reached), obtaining the ETS capacity, ETS $\max _{\text {, with }}$ convergent electron flow through CI+II. Electron input into the Q-junction through complex II (CII) alone was subsequently induced by inhibition of CI by rotenone (Rot; $0.5 \mu \mathrm{M}$ ). Finally, residual oxygen consumption (ROX) was obtained by addition of antimycinA $(2.5 \mu \mathrm{M})$ to block electron transfer through complex III (CIII]). Oxygen fluxes were corrected by subtracting ROX from each measured mitochondrial steady-state.

Integrity of the outer mitochondrial membrane was tested by adding $10 \mu \mathrm{M}$ cytochrome $\mathrm{c}$ after ADP in the presence of substrates feeding electrons into CI. Injury of the outer mitochondrial membrane leads to loss of cytochrome $\mathrm{c}$ from the mitochondria, and to significant stimulation of respiration following addition of exogenous cytochrome $\mathrm{c}$ to the respiration medium.

Oxygen flux was expressed as tissue mass-specific respiration (per $\mathrm{mg}$ ), or as Flux Control Ratios (FCR), with internal normalization for maximum ETS capacity.

\section{Statistical analysis}

Respirometry measurements were performed at least in duplicate for each protocol. The oxygen flux and FCR are presented as mean \pm SD for the 4 groups, i.e. Cont fit, Contob, Met Lam and Infl Lam. A levene's test was used for all the dependent variables to test the equality of variance then a two-way analysis of variance was used for each dependent variable taking into account two qualitative factors: 
score and laminitis. Furthermore, a Student $\mathrm{T}$ test were used when the variances are equal to compare the mean oxygen flux values of $\mathrm{CI}, \mathrm{CI}$ + II, ETS $_{\max }$ and CII alone between the Cont fit group and the Infl lam group and between the Contob group and the Met lam group. The BSC were compared between the groups by a Mann-Whitney test. A value of $\mathrm{p}<0.05$ was considered as level of significance (MedCalc; MedCalc Software, Version 12.7.7.; Ostend, Belgium).

\section{Results}

The comparison of the BSC measured in each group showed significant differences between the Cont fit and the Contob groups and between the Infl Lam and the Met Lam groups.

The figure 1 illustrates oxygraphic records obtained on one healthy horse and two laminitic horses (Metabolic Laminitis, Inflammatory Laminitis).

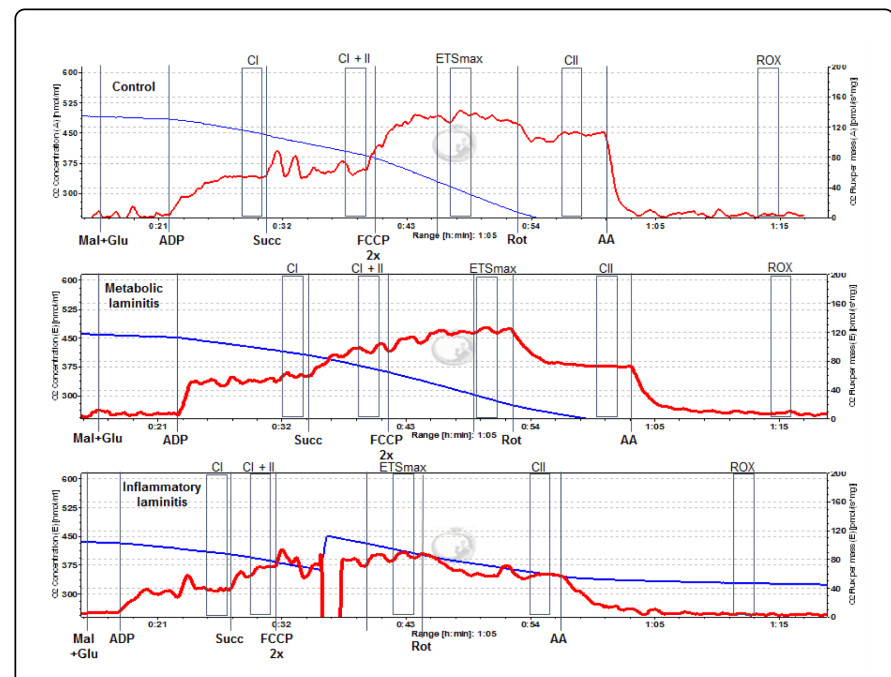

Figure 1: Example of HRR curves. Illustration of the curves obtained via the Oroboros oxygraph software [DatLab 4.0, OROBOROS DatLab software, Innsbruck, Austria] for one horse of each group [Control, Metabolic laminitis and Inflammatory laminitis]. The blue line represents the oxygen concentration in the medium $[\mathrm{pmol} / \mathrm{ml}]$ and the red line represents the slope of the blue line, thus the oxygen flux expressed as tissue mass-specific respiration [pmol oxygen $/ \mathrm{sec}^{\star} \mathrm{mg}$ of permeabilized fibers]. The vertical lines represent the different substrates/inhibitors/ uncouplers added to the oxygraph chambers. The black rectangles represent the selected time laps during which CI [Complex I], CI + II [Complex I and 2], maximal respiratory [ETSmax] and CII [Complex II] capacities were calculated by DatLab ${ }^{\mathrm{Tm}}$.

The Cont fit horses showed a mean tissue mass-specific respiration (per mg of muscle) of $58.79( \pm 12.56) \mathrm{pmol} \mathrm{O}_{2} / \mathrm{sec}$ with $\mathrm{CI}$ substrates (Table 1). Their maximal OXPHOS and ETS capacities with physiological CI+II substrates were $116.37( \pm 18.89)$ and 139.65 $( \pm 19.38) \mathrm{pmol} \mathrm{O}_{2} / \mathrm{sec}$ respectively. For the $\mathrm{CII}$ alone, the tissue massspecific respiration was $96.13( \pm 12.82) \mathrm{pmol} \mathrm{O}_{2} / \mathrm{sec}$. The ContOb horses showed a mean tissue mass-specific respiration (per $\mathrm{mg}$ of muscle) of $51.60( \pm 13.98) \mathrm{pmol} \mathrm{O}_{2} / \mathrm{sec}$ with CI substrates (Table 1). Their maximal OXPHOS and ETS capacities with physiological CI+II substrates were $102.76( \pm 23.46)$ and $119.76( \pm 24.27) \mathrm{pmol} \mathrm{O}_{2} / \mathrm{sec}$ respectively. For the $\mathrm{CII}$ alone, the tissue mass-specific respiration was
$81.85( \pm 16.79)$ pmol $\mathrm{O}_{2} / \mathrm{sec}$. Compared to the cont fit horses, the contob horses showed a significant decrease of ETS capacities and CII alone.

The two-way analysis of variance to test the effect of the two qualitative factors (Score and laminitis) revealed that any dependent variable was significantly affected by the body score index. However, a significant effect of the factor "laminitis" was observed for CI, CI+II, ETS capacities, CII alone. Otherwise, when FCR was applied only the CII was significantly different between the horses affected or not by the disease. The T-test analysis comparing the ContOb group to the Met Lam group showed a significant decrease of the oxygen flux for CI $+\mathrm{CII}$ and CII alone. Compared to Cont Fit horses, the Infl Lam group showed a significant decrease of the oxygen flux for CI+CII, ETS capacities and CII alone.

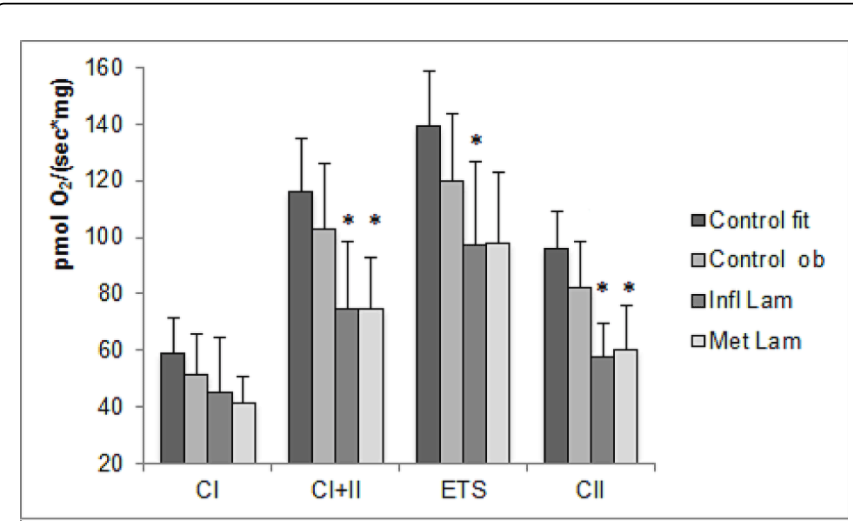

Figure 2: Tissue mass-specific respirations. Tissue mass-specific respirations [mean $\pm \mathrm{SD}$ of oxygen flux per mg of muscle; pmol $\mathrm{O}_{2}{ }^{\star} \mathrm{sec}-1^{\star} \mathrm{mg}-1$ ] of control non obese [Cont fit], control obese [ContOb], metabolic laminitis [Met Lam] and inflammatory laminitis [Infl Lam] horses. CI: Oxygen flux via mitochondrial complex I; CI+II: Oxygen flux via mitochondrial complex I and II; ETS: maximal respiratory capacity after total uncoupling with carbonyl cyanide 4-[trifluoromethoxy] phenylhydrazone; CII: Oxygen flux via mitochondrial complex II alone. ${ }^{*} \mathrm{p}<0.05$ Inf Lam vs. Control non $\mathrm{Ob}$ or Met Lam vs. ControlOb

The SUIT protocol allowed us to also establish the FCR for each group of horses (Figure 3). Cont fit horses used $41.68( \pm 5.08) \%$ and $68.99( \pm 7.21) \%$ of their maximal ETS capacity via respectively CI and CII alone. When substrates for CI and CII are available (CI+II), they used $83.45( \pm 9.46) \%$ of their maximal ETS capacity. Similar values are observed in the control obese group (CI: $43.11 \pm 6.9$; CII: $68.87 \pm 9.13$; CI+CII: $86.16 \pm 12.32)$. The CI+CII values represented the maximal proportion of their respiratory capacity that mitochondria are effectively able to use for producing ATP, and thus, energy. Notwithstanding, via CI, Met Lam or Infl Lam horses did not use a significant greater proportion of their respective maximal ETS capacity than Control horses. On the contrary, the 2 pathological groups used a significant lower part of their maximal ETS capacity via CII: 61.72 ( \pm $4.85) \%$ for Met Lam and only 59.29 ( \pm 9.33) \% for Infl Lam. However,

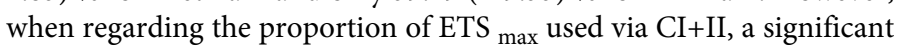
reduction was only observed for Met Lam horses as compared to the Contob group.Thus, they were able to use only $75.94( \pm 8.1) \%$ of their maximal respiratory capacity. A similar non-significant trend was also observed in the Infl Lam group. 
Page 5 of 7

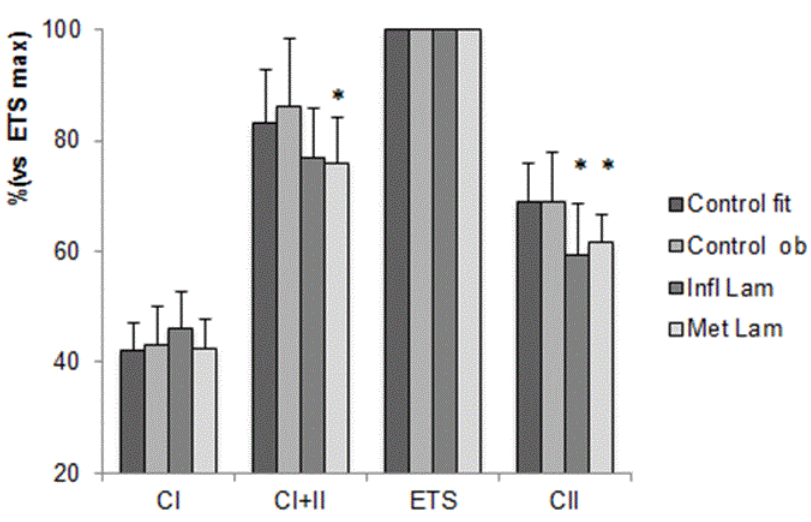

Figure 3: Flux control ratios. Flux control ratios [mean $\pm \mathrm{SD}]$ of control non obese, control obese, metabolic laminitis [Met Lam] and inflammatory laminitis [Infl Lam] horses, standardized for their respective maximal respiratory capacities [ETSmax $=100 \%$ for each experimental group]. CI: part of ETSmax used via mitochondrial complex I; CI+II: part of ETSmax used via mitochondrial complex I and II; ETS max: maximal respiratory capacity; CII: part of ETSmax used via mitochondrial complex II.* $\mathrm{p}<0.05$ Met Lam vs. ContOb or Infl Lam vs. Cont fit

\section{Discussion}

The results of the present study confirm the hypothesis that significant reduction of muscle mitochondrial respiration is observed during the acute phase of laminitis. The reduction of OXPHOS may result in a depletion of the cells' ATP content. This is especially true if the glycolytic pathway cannot compensate for the deficient ATP. Laminitis is considered as a systemic disease with clinical signs localized in the foot. Even where mitochondrial dysfunction has not been demonstrated in the foot, energy deficiency has been suggested as an explanation for the disruption of the hemidesmosomes leading to the failure of the dermal-epidermal interface [32].

An energy deficit can arise either from a reduction in the supply of oxygen and/or nutrients or from impaired mitochondrial function in itself. The HRR procedure with supplemented substrates (glutamate, malate and ADP) performed on permeabilized muscle micro-biopsies to measure OXPHOS and ETS capacities demonstrated that the mitochondrial respiratory chain is clearly affected.

Several authors consider that the OXPHOS and maximal ETS capacity are influenced by the physical activity and the body mass index of the subject $[33,34]$. Votion et al. showed lower respirometric parameters in overweight and untrained horses than in competition horses [29]. Thus, two groups of control horses were included in this study with the aim to clearly identify the effects of a high BSC. The two way analysis of variance to test the BSC was not significant and the interaction between the two factors (BSC and laminitis) was not significant for each tested variables. Nevertheless, using the T-test, we observed a significant reduction of maximal ETS capacity and CII alone in the obese horses compared to the horses with a normal BSC. But when oxygen flux was expressed as tissue mass-specific respiration (per mg), or as flux control ratios, with internal normalization for maximum ETS capacity (FCR), no significant difference were observed between the two control groups. Matching of breed and level of training was realized for the normal BSC groups, in order to obtain an adequate control group for the inflammatory laminitis group.

Nevertheless, the oxygen flux for CI+CII, CII alone were significantly reduced in both acute laminitis groups compared to healthy horses and maximal ETS capacity especially in the Infl Lam group.

The differences between the laminitis horses and the control horses having a high BSC could be related to a certain level of metabolic syndrome. Indeed, equine metabolic syndrome is characterized by obesity and local adiposity coupled with evidence of recurrent laminitis [35]. These authors observed that a marked increase in neutrophil reactive oxygen species production upon phagocytosis was observed in horses with metabolic syndrome that was closely correlated to the blood insulin concentration. Insulin resistance is currently observed in horses suffering from metabolic syndrome. Based on the results of this study, it would have been interesting to dose insulin in our patients. However, to confirm insulin resistance, it is also necessary to show abnormal glycemic and insulinemic responses to oral or IV glucose and/or insulin challenges. These tests would have been ethically unacceptable on clinical cases, taking into account the risks of aggravating the disease.

When internal normalization for maximum ETS capacity (FCR) was applied, we observed clearly that the complex II was principally affected. Mitochondrial complex II oxidizes succinate to fumarate as part of the Krebs cycle and reduces ubiquinone in the electron transport system. Succinate-driven oxidation via complex II may contribute significantly to high rates of production of Reactive Oxygen Species (ROS) by mitochondria [36,37].

A Complex II defect was accompanied by a biphasic increase of ROS and concurrent glutathione oxidation, both concomitant to a decrease of the ATP cellular level [38]. High ROS generation is considered as a key point in human patients affected by metabolic syndrome and insulin resistance [39]. The implication of ROS in the pathogenesis of laminitis has already been suggested [40,41]. Moreover, activated neutrophils generate ROS and release oxidant enzyme such as myeloperoxidase and proteases including elastase and matrix metalloproteinase 9. In a model of muscle cells in culture, it was demonstrated that myeloperoxidase increased significantly the ROS amount generated by anoxia and reoxygenation cycles [42]. Thus, the production of ROS in lamina could be explained by neutrophil activation with the presence of myeloperoxidase in the lamina and/or by the mitochondrial dysfunction.

In this study, the muscle mitochondrial dysfunction was observed independently of the key factors responsible of the laminitis [metabolic or inflammation]. If the same dysfunction occurs in the foot lamina, it might be possible that the ATP available to maintain the hemidesmosome activity becomes insufficient, once the alteration of the OXPHOS reaches a certain level, and that this would occur independently of the etiology. In other words, a «mitochondrial dysfunction threshold» would be reached to trigger the pathology.

To improve the comprehension of this pathophysiological mechanism, mitochondrial respiration and ATP levels should be studied on muscular biopsies from experimental laminitis induced by $\mathrm{CHO}, \mathrm{BWE}, \mathrm{OF}$ administration or hyperinsulinemic/euglycemic clamp model.

The mitochondrial function may be measured directly on lamina biopsies obtained immediately following euthanasia when the horses 
show an OBEL 1 grade. Mitochondrial respiration may also be measured on muscle micro-biopsies following the administration of the triggering factor.

Since samples were obtained from clinical cases, standardization was not possible and factors such as the time point of the micro-biopsy related to the onset of the laminitis, the gravity of the disease, and treatment before referral to the clinic may have influenced the results. These factors may partly explain the wide standard deviation observed.

\section{Conclusion}

This clinical study shows for the first time a significant decrease of muscle mitochondrial oxidative phosphorylation in horses affected by acute laminitis when compared to the values obtained from fit or obese healthy horses. Targeting the mitochondria in the foot should be considered as essential for a better understanding of the physiopathology and for new treatment strategies, with the aim to maintain and to support the mitochondrial function before the « mitochondrial dysfunction threshold » is reached and failure of the dermal-epidermal interface occurs.

\section{Conflict of interest statement}

The authors certify that there is no conflict of interest with any financial organization regarding the material discussed in the manuscript.

\section{Acknowledgements}

The authors thank Ariane Niesten and Jennifer Romainville for their technical assistance for the high resolution respirometry measurements.

\section{References}

1. Katz LM, Bailey SR (2012) A review of recent advances and current hypotheses on the pathogenesis of acute laminitis. Equine Vet J 44: 752-761.

2. Menzies-Gow NJ, Katz LM, Barker KJ, Elliott J, De Brauwere MN, et al. (2010) Epidemiological study of pasture-associated laminitis and concurrent risk factors in the South of England. Vet Rec 167: 690-694.

3. Moore JN, Belknap JK (2009) You say lamellae, I say laminae. Let's call ... An overview of the Havemeyer workshop on laminitis pathophysiology. Vet ImmunolImmunopathol 129: 149-150.

4. Parsons CS, Orsini JA, Krafty R, Capewell L, Boston R (2007) Risk factors for development of acute laminitis in horses during hospitalization: 73 cases (1997-2004]. Journal of American Veterinary Medicine Association 230: $885-889$.

5. Wylie CE, Collins SN, Verheyen KL, Newton JR (2013) Risk factors for equine laminitis: a case-control study conducted in veterinary-registered horses and ponies in Great Britain between 2009 and 2011. Vet J 198: 57-69.

6. de la Rebière de Pouyade G, Serteyn D (2011) The role of activated neutrophils in the early stage of equine laminitis. Vet J 189: 27-33.

7. Weiss DJ, Evanson OA, McClenahan D, Fagliari JJ, Jenkins K (1997) Evaluation of platelet activation and platelet-neutrophil aggregates in ponies with alimentary laminitis. Am J Vet Res 58: 1376-1380.

8. Black SJ, Lunn DP, Yin C, Hwang M, Lenz SD, et al. (2006) Leukocyte emigration in the early stages of laminitis. Vet ImmunolImmunopathol 109: 161-166.

9. Hurley DJ, Parks RJ, Reber AJ, Donovan DC, Okinaga T, et al. (2006) Dynamic changes in circulating leukocytes during the induction of equine laminitis with black walnut extract. Vet ImmunolImmunopathol 110: 195-206.

10. Loftus JP, Belknap JK, Stankiewicz KM, Black SJ (2007) Laminar xanthine oxidase, superoxide dismutase and catalase activities in the prodromal stage of black-walnut induced equine laminitis. Equine Vet J 39: 48-53.

11. Tadros EM, Frank N, Newkirk KM, Donnell RL, Horohov DW (2012) Effects of a "two-hit" model of organ damage on the systemic inflammatory response and development of laminitis in horses. Vet ImmunolImmunopathol 150: 90-100.

12. Kwon S, Moore JN, Robertson TP, Hurley DJ, Wagner B, et al. (2013) Disparate effects of LPS infusion and carbohydrate overload on inflammatory gene expression in equine laminae. Vet ImmunolImmunopathol 155: 1-8.

13. Johnson PJ, Tyagi SC, Katwa LC, Ganjam VK, Moore LA, et al. (1998) Activation of extracellular matrix metalloproteinases in equine laminitis. Vet Rec 142: 392-396.

14. van Eps AW, Pollitt CC (2006) Equine laminitis induced with oligofructose. Equine Vet J 38: 203-208.

15. Riggs LM, Franck T, Moore JN, Krunkosky TM, Hurley DJ, et al. (2007) Neutrophil myeloperoxidase measurements in plasma, laminar tissue, and skin of horses given black walnut extract. Am J Vet Res 68: 81-86.

16. de la Rebière de Pouyade G, Riggs LM, Moore JN, Franck T, DebyDupont G et al. (2010) Equine neutrophil elastase in plasma, laminar tissue and skin of horses administered black walnut heartwood extract. Veterinary Immunology and Immunopathology 13 : 181-187.

17. Franck T, Votion DM, Ceusters J, De La Rebière de Pouyade G, Mouithys-Mickalad A, et al. (2010) Specific immuno-extraction followed by enzymatic detection (SIEFED) of myeloperoxidase and mitochondrial complex I in muscular microbiopsies: preliminary results in endurance horses. Equine Vet J Suppl : 296-302.

18. Ceusters JD, Mouithys-Mickalad AA, Franck TJ, Derochette S, Vanderplasschen A, et al. (2013) Effect of myeloperoxidase and anoxia/ reoxygenation on mitochondrial respiratory function of cultured primary equine skeletal myoblasts. Mitochondrion 13: 410-416.

19. Johnson PJ, Messer NT, Ganjam VK (2004) Cushing's syndromes, insulin resistance and endocrinopathic laminitis. Equine Vet J 36: 194-198.

20. Geor R, Frank N (2009) Metabolic syndrome-From human organ disease to laminar failure in equids. Vet ImmunolImmunopathol 129: 151-154.

21. de Laat MA, McGowan CM, Sillence MN, Pollitt CC (2010) Equine laminitis: induced by $48 \mathrm{~h}$ hyperinsulinaemia in Standardbred horses. Equine Vet J 42: 129-135.

22. Karikoski NP1, McGowan CM2, Singer ER2, Asplin KE3, Tulamo RM4, et al. (2014) Pathology of Natural Cases of Equine Endocrinopathic Laminitis Associated With Hyperinsulinemia. Vet Pathol .

23. Gauff F1, Patan-Zugaj B, Licka TF (2013) Hyperinsulinaemia increases vascular resistance and endothelin-1 expression in the equine digit. Equine Vet J 45: 613-618.

24. Lowell BB, Shulman GI (2005) Mitochondrial dysfunction and type 2 diabetes. Science 307: 384-387.

25. Kim JA, Wei Y, Sowers JR (2008) Role of mitochondrial dysfunction in insulin resistance. Circ Res 102: 401-414.

26. Cheng $\mathrm{Z}$, Tseng $\mathrm{Y}$, White MF (2010) Insulin signaling meets mitochondria in metabolism. Trends EndocrinolMetab 21: 589-598.

27. Abhijit S, Bhaskaran R, Narayanasamy A, Chakroborty A, Manickam N, et al. (2013) Hyperinsulinemia-induced vascular smooth muscle cell (VSMC) migration and proliferation is mediated by converging mechanisms of mitochondrial dysfunction and oxidative stress. Mol Cell Biochem 373: 95-105.

28. Gnaiger E (2009) Capacity of oxidative phosphorylation in human skeletal muscle: new perspectives of mitochondrial physiology. Int J Biochem Cell Biol 41: 1837-1845.

29. Votion DM, Gnaiger E, Lemieux H, Mouithys-Mickalad A, Serteyn D (2012) Physical fitness and mitochondrial respiratory capacity in horse skeletal muscle. PLoS One 7: e34890. 
Citation: Serteyn D, Pouyade G R, Sandersen C, Salciccia A, Grulke S, et al (2014) Muscle Mitochondrial Dysfunction in Horses Affected by Acute Laminitis. Bioenergetics 3: 1000120. doi:dx.doi.org/10.4172/2167-7662.1000120

Page 7 of 7

30. Arnaud G, Dubroeucq H, Rivot D (1997) Notation de l'étatcorporel des chevaux de selle et de sport. Inra, Institut du cheval, Institut de l'Elevage, Paris.

31. Frank N, Geor RJ, Bailey SR, Durham AE, Johnson PJ; American College of Veterinary Internal Medicine (2010) Equine metabolic syndrome. J Vet Intern Med 24: 467-475.

32. French KR, Pollitt CC (2004) Equine laminitis: loss of hemidesmosomes in hoof secondary epidermal lamellae correlates to dose in an oligofructose induction model: an ultrastructural study. Equine Veterinary Journal 36: 230-235.

33. Bakkman L, Sahlin K, Holmberg HC, Tonkonogi M (2007) Quantitative and qualitative adaptation of human skeletal muscle mitochondria to hypoxic compared with normoxic training at the same relative work rate. ActaPhysiol (Oxf) 190: 243-251.

34. Hütter E, Skovbro M, Lener B, Prats C, Rabøl R, et al. (2007) Oxidative stress and mitochondrial impairment can be separated from lipofuscin accumulation in aged human skeletal muscle. Aging Cell 6: 245-256.

35. Holbrook TC, Tipton T, McFarlane D (2012) Neutrophil and cytokine dysregulation in hyperinsulinemic obese horses. Vet ImmunolImmunopathol 145: 283-289.

36. Quinlan CL, Orr AL, Perevoshchikova IV, Treberg JR, Ackrell BA, et al. (2012) Mitochondrial complex II can generate reactive oxygen species at high rates in both the forward and reverse reactions. J BiolChem 287: $27255-27264$.
37. Moreno-Sánchez R, Hernández-Esquivel L, Rivero-Segura NA, MarínHernández A, Neuzil J, et al. (2013) Reactive oxygen species are generated by the respiratory complex II--evidence for lack of contribution of the reverse electron flow in complex I. FEBS J 280: 927-938.

38. Byun HO, Kim HY, Lim JJ, Seo YH, Yoon G (2008) Mitochondrial dysfunction by complex II inhibition delays overall cell cycle progression via reactive oxygen species production. J Cell Biochem 104: 1747-1759.

39. Houstis N, Rosen ED, Lander ES (2006) Reactive oxygen species have a causal role in multiple forms of insulin resistance. Nature 440: 944-948.

40. Loftus JP, Black SJ, Pettigrew A, Abrahamsen EJ, Belknap JK (2007) Early laminar events involving endothelial activation in horses with black walnut- induced laminitis. Am J Vet Res 68: 1205-1211.

41. Treiber K, Carter R, Gay L, Williams C, Geor R (2009) Inflammatory and redox status of ponies with a history of pasture-associated laminitis. Vet ImmunolImmunopathol 129: 216-220.

42. Ceusters JD, Mouithys-Mickalad AA, de la Rebière de Pouyade G, Franck TJ, Votion DM, et al. (2012) Assessment of reactive oxygen species production in cultured equine skeletal myoblasts in response to conditions of anoxia followed by reoxygenation with or without exposure to peroxidases. Am J Vet Res 73: 426-434. 